\title{
Ubiquitous suppression of the nodal coherent spectral weight in Bi-based cuprates
}

\author{
M. Zonno ${ }^{1},{ }^{1,2,{ }^{*}}$ F. Boschini ${ }^{1},{ }^{1,2, \dagger}$ E. Razzoli, ${ }^{1,2,3}$ S. K. Y. Dufresne ${ }^{\circledR},{ }^{1,2}$ M. Michiardi, ${ }^{1,2,4}$ M. X. Na ${ }^{\circledR},{ }^{1,2}$ T. M. Pedersen, ${ }^{5}$ \\ S. Gorovikov, ${ }^{5}$ S. Gonzalez $\odot,{ }^{6}$ G. Di Santo $\odot,{ }^{6}$ L. Petaccia $\odot,{ }^{6}$ M. Schneider $\odot,{ }^{1}$ D. Wong, ${ }^{1}$ P. Dosanjh, ${ }^{1}$ Y. Yoshida, ${ }^{7}$ \\ H. Eisaki ${ }^{7}{ }^{7}$ R. D. Zhong, ${ }^{8}$ J. A. Schneeloch, ${ }^{8}$ G. D. Gu, ${ }^{8}$ A. K. Mills ${ }^{1},{ }^{1,2}$ S. Zhdanovich ${ }^{\circledR},{ }^{1,2}$ G. Levy ${ }^{\circledR},{ }^{1,2}$ \\ D. J. Jones $\odot{ }^{1,2}$ and A. Damascelli ${ }^{1}{ }^{1,2, \$}$ \\ ${ }^{1}$ Quantum Matter Institute, University of British Columbia, Vancouver, British Columbia, Canada, V6T $1 Z 4$ \\ ${ }^{2}$ Department of Physics \& Astronomy, University of British Columbia, Vancouver, British Columbia, Canada, V6T $1 Z 1$ \\ ${ }^{3}$ Swiss FEL, Paul Scherrer Institut, 5232 Villigen PSI, Switzerland \\ ${ }^{4}$ Max Planck Institute for Chemical Physics of Solids, Nöthnitzer Straße 40, Dresden 01187, Germany \\ ${ }^{5}$ Canadian Light Source, Inc.44 Innovation Boulevard, Saskatoon, Saskatchewan, Canada, S7N 2V3 \\ ${ }^{6}$ Elettra Sincrotrone Trieste, Strada Statale 14 km 163.5, 34149 Trieste, Italy \\ ${ }^{7}$ National Institute of Advanced Industrial Science and Technology (AIST), Tsukuba 305-8568, Japan \\ ${ }^{8}$ Condensed Matter Physics and Materials Science, Brookhaven National Laboratory, Upton, New York 11973-5000, USA
}

(Received 9 September 2020; revised 9 March 2021; accepted 11 March 2021; published 7 April 2021)

\begin{abstract}
High-temperature superconducting cuprates exhibit an intriguing phenomenology for the low-energy elementary excitations. In particular, an unconventional temperature dependence of the coherent spectral weight (CSW) has been observed in the superconducting phase by angle-resolved photoemission spectroscopy (ARPES), both at the antinode where the $d$-wave paring gap is maximum, as well as along the gapless nodal direction. Here, we combine equilibrium and time-resolved ARPES to track the temperature-dependent meltdown of the nodal CSW in Bi-based cuprates with unprecedented sensitivity. We find the nodal suppression of CSW upon increasing temperature to be ubiquitous across single- and bi-layer Bi cuprates, and uncorrelated to superconducting and pseudogap onset temperatures. We quantitatively model both the lineshape of the nodal spectral features and the anomalous suppression of CSW within the Fermi-liquid framework, establishing the key role played by the normal state electrodynamics in the description of nodal quasiparticles in superconducting cuprates.
\end{abstract}

DOI: 10.1103/PhysRevB.103.155109

\section{INTRODUCTION}

Copper-oxide high- $T_{c}$ superconductors host a variety of emerging and competing quantum phases. On the one hand, their low temperature physics is mainly characterized by unconventional $d$-wave superconductivity (SC); on the other hand, the normal state is actually a "strange metal" exhibiting various complex phenomena, such as pseudogap physics and charge density wave order [1-4]. Since the discovery of cuprates, the existence (or lack thereof) of well-defined quasiparticles in the normal state, as well as their temperature and doping dependence, have been the subject of intense debate. The characteristic onset temperature $T_{c}$ has been associated not only with the SC phase transition, but also with the emergence of coherent quasiparticles $[5,6]$. In this regard, specific insight has come from angle-resolved photoemission spectroscopy (ARPES), either at equilibrium or in pumpprobe configuration, which provides access to the low-energy electronic structure and its dynamics [5-15]. In particular,

\footnotetext{
*mzonno@phas.ubc.ca

†Present address: Centre Énergie Matériaux Télécommunications, Institut National de la Recherche Scientifique, Varennes, Québec J3X 1S2, Canada.

${ }^{\ddagger}$ damascelli@physics.ubc.ca
}

an unconventional temperature dependence of the coherent spectral weight (CSW) for antinodal quasiparticles has been reported by ARPES studies on $\mathrm{Bi}_{2} \mathrm{Sr}_{2} \mathrm{CaCu}_{2} \mathrm{O}_{8+\delta}$ ( $\mathrm{Bi2212}$ ); this was described either as a manifestation of the $c$-axis superfluid density, or as a testament to the different role played by the quasiparticle coherence in the emergence of SC in underdoped versus overdoped regimes $[8,9]$.

Towards unveiling the detailed evolution of quasiparticle weight and lifetime from normal to SC state, the study of the antinodal region of cuprates is hindered by the interplay of several intertwined contributions, such as SC gap, pseudogap, charge order, as well as strong band renormalization. More promising is, in principle, the exploration of the gapless and more pristine nodal direction. Similarly to what was reported at the antinode, a time-resolved ARPES (TR-ARPES) study of optimally doped $\mathrm{Bi} 2212$ has suggested a direct relation between nodal CSW and the SC condensate [13]. However, a recent theoretical work questioned such a direct link, proposing instead the key role of a different competing order [16]. As a consequence, for both nodal and antinodal regions of the $\mathrm{CuO}_{2}$ plane electronic structure, a comprehensive and conclusive description of the evolution of CSW across the superconducting-to-normal-state phase transition is still missing.

In laying the basis for discussing the temperature dependence ( $T$ dependence) of CSW in cuprates, we remark 

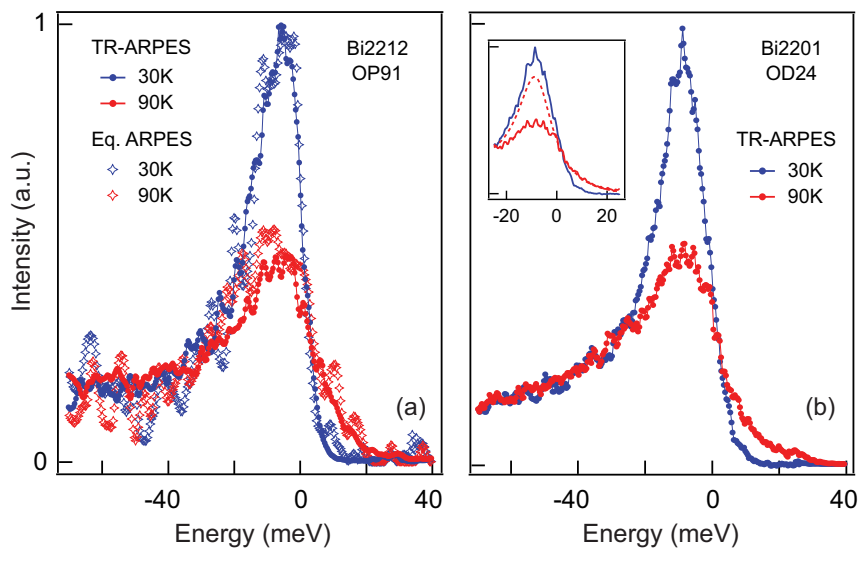

FIG. 1. (a) Energy distribution curves (EDCs) at the Fermi momentum $\mathbf{k}_{\mathrm{F}}$ along the nodal direction of Bi2212-OP91 for $30 \mathrm{~K}$ and $90 \mathrm{~K}$, as measured by TR-ARPES $(h v=6.2 \mathrm{eV})$ and equilibrium ARPES $(h v=27 \mathrm{eV})$. (b) Nodal EDCs at $\mathbf{k}_{\mathrm{F}}$ for Bi2201-OD24 acquired by TR-ARPES. Inset: Comparison between the same experimental data (solid lines) and the EDC obtained from the $30 \mathrm{~K}$ data via the thermal broadening of the Fermi-Dirac distribution at $90 \mathrm{~K}$ (dashed line). All EDCs have been deconvoluted from the energy resolution broadening via the Lucy-Richardson algorithm [15,20,21] [TR-ARPES energy resolution is $11 \mathrm{meV}$ and $18 \mathrm{meV}$ for panels (a) and (b), respectively; equilibrium ARPES energy resolution is $5.3 \mathrm{meV}]$.

that the many-body renormalization of lifetime and pole structure is captured by the complex electron self-energy $\Sigma(\omega, T)=\Sigma^{\prime}(\omega, T)+i \Sigma^{\prime \prime}(\omega, T)$, where the momentum dependence is commonly neglected. ARPES experiments provide access to $\Sigma(\omega, T)$ by measuring the single-particle removal spectral function $A(\mathbf{k}, \omega, T)$, with its corresponding many-body self-energy corrections [1]. In the hypothetical scenario of a noninteracting system, $A(\mathbf{k}, \omega, T)$ collapses to a series of delta functions whose area is not expected to vary as a function of temperature. However, in the presence of electron interactions $\Sigma(\omega, T)$ may lead to the broadening and redistribution of the spectral weight within the momentum-energy phase space. Therefore, one may argue that the observed $T$ dependence of CSW for a welldefined momentum-specifically the Fermi momentum $\mathbf{k}_{\mathrm{F}}$, $\operatorname{CSW}\left(\mathbf{k}_{\mathrm{F}}, T\right)=\int_{-\infty}^{\infty} A\left(\mathbf{k}_{\mathrm{F}}, \omega, T\right) d \omega$-is determined by the underlying electron interactions encoded in $\Sigma(\omega, T)$ (note that our discussion is centered solely on the $d_{x^{2}-y^{2}}$ antibonding band, see Appendix B).

In this work, we establish the role played by the normalstate self-energy to the integrity of quasiparticles by tracking the $T$ dependence of both the $\omega=0$ and energy-integrated nodal spectral function at $\mathbf{k}_{\mathrm{F}}$. This is achieved for both singleand bi-layer Bi cuprates at various dopings by employing TR-ARPES, which offers an enhanced sampling and signalto-noise for the $T$ dependence of the nodal spectra. This approach, corroborated by conventional equilibrium ARPES, reveals a ubiquitous suppression of nodal CSW that bears no relation to $T_{c}$, but instead directly stems from the $\omega$ and $T$ dependence of the complex self-energy. By analyzing the data in terms of existing models, such as Fermi liquid (FL) [17], marginal Fermi liquid (MFL) [18], and $T^{3}$ [19] models, we find that solely the FL framework reproduces comprehensively the $T$ dependence of the nodal CSW, as well as the overall nodal quasiparticle phenomenology encoded in the lineshape and amplitude of energy and momentum distribution curves (EDCs and MDCs).

\section{EXPERIMENTAL STRATEGY}

Equilibrium and time-resolved ARPES measurements were performed on high quality $\mathrm{Bi}_{2} \mathrm{Sr}_{2-x} \mathrm{La}_{x} \mathrm{CuO}_{6+\delta}(\mathrm{Bi} 2201)$ and $\mathrm{Bi}_{2} \mathrm{Sr}_{2} \mathrm{CaCu}_{2} \mathrm{O}_{8+\delta}(\mathrm{Bi} 2212)$ single crystals at various doping levels along the nodal $\bar{\Gamma}-\bar{Y}$ direction (details in Appendix A). Figure 1 shows EDCs at $\mathbf{k}_{\mathrm{F}}$ along the nodal direction, for two different temperatures of optimally doped Bi2212 $\left(T_{c}=91 \mathrm{~K}\right.$, OP91 $)$ and overdoped Bi2201 $\left(T_{c}=\right.$ $24 \mathrm{~K}$, OD24) - energy resolution broadening was removed via the Lucy-Richardson algorithm [15,20,21]. For both compounds, we observe a clear suppression of CSW close to the Fermi energy $\left(E_{\mathrm{F}}\right)$, as the temperature increases from 30 to $90 \mathrm{~K}$. As an alternative approach to standard $T$ dependent ARPES, in this work we employ TR-ARPES to meticulously track the temperature-dependent modifications of the low-energy nodal spectral features by tuning the transient electronic temperature $T_{\mathrm{e}}$ via optical pumping. Due to the strong electron interactions underlying the cuprates, quasiparticles excited via near-infrared light thermalize on an ultrashort timescale (i.e., $\sim 100 \mathrm{fs}$; note that a similar timescale was reported also for the photodoping effects [22]), entering a state of quasiequilibrium where an effective electronic temperature can be defined at each pumpprobe delay $[13,23,24]$. This method allows one to directly link the transient electronic temperature to the progression of the ARPES spectral features, effectively performing a $T$-dependent measurement with minimal repercussions from surface degradation and sample drifting. To convert the measured time dependence of our data into a temperature evolution, we fit the Fermi edge width of the momentumintegrated EDCs along the nodal direction, as illustrated in
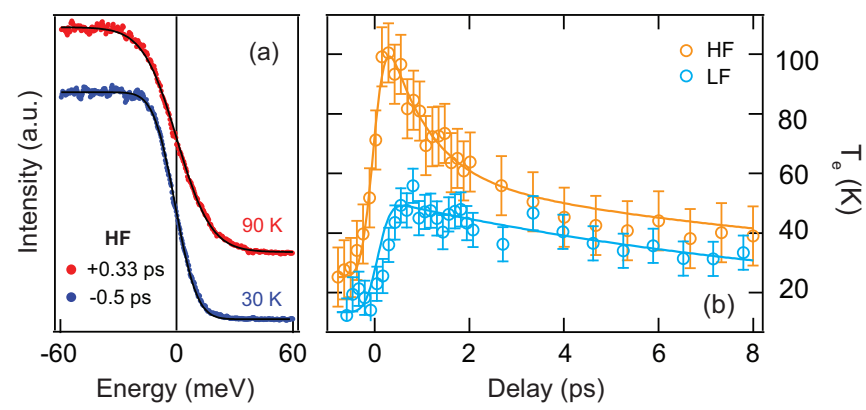

FIG. 2. (a) Momentum-integrated EDCs along the nodal direction before $(-0.5 \mathrm{ps})$ and after $(+0.33 \mathrm{ps})$ the pump excitation for Bi2201-OD24. Fermi-Dirac distribution fits are shown as solid black lines; temperature values are defined by a $99 \%$-confidence-interval. (b) Transient electronic temperature $T_{\mathrm{e}}$ obtained by fitting the Fermi edge width along the nodal direction of Bi2201-OD24, for two different pump fluences $\left(12 \mu \mathrm{J} / \mathrm{cm}^{2}\right.$, LF; $90 \mu \mathrm{J} / \mathrm{cm}^{2}$, HF). The solid lines represent (single-) double-exponential decay fit to (LF) HF data. Error bars in panel (b) reflect the systematic errors associated with the experiment and the number of averaging cycles acquired for each fluence. All data acquired via TR-ARPES at $6.2 \mathrm{eV}$. 
Fig. 2 for overdoped Bi2201-OD24. Note that the resulting transient electronic temperature $T_{\mathrm{e}}$ can be phenomenologically described by an exponential-decay function [Fig. 2(b), solid line].

Although this ultrafast approach ensures equivalent experimental conditions for each temperature (e.g., same probed spot on the sample's surface and momentum-space position), it might raise doubts on (i) whether the probed behavior is an exclusive property of the transient state and (ii) how different transient lattice and electronic temperatures may complicate the data interpretation. To address these points, we compare in Fig. 1(a) the nodal EDCs at $\mathbf{k}_{\mathrm{F}}$ measured by TR-ARPES at $6.2 \mathrm{eV}$ and conventional equilibrium ARPES at $27 \mathrm{eV}$. The good agreement between the two different probing methods indicates that the suppression of CSW is inherently related to the electronic temperature of the system, thus validating the TR-ARPES approach as our main experimental strategy throughout the entire work, as well as suggesting the predominant role played by electron-electron interactions in the suppression of nodal CSW. Moreover, the data in Fig. 1(a) exclude the explicit breaking of the "sudden-approximation" with low-energy photons [25] (also note that only the antibonding state is probed using both $6.2 \mathrm{eV}$ and $27 \mathrm{eV}$, and nonlinear effects in the photoelectrons' detection [26] have been ruled out; details in Appendix B and C). Finally, as illustrated in the inset of Fig. 1(b), the mere thermal broadening of the Fermi-Dirac distribution at $90 \mathrm{~K}$ does not account for the observed $T$ dependence of the experimental data.

\section{RESULTS AND ANALYSIS}

Having defined the experimental strategy, we now examine the temperature-dependent modifications of the nodal spectral features of various Bi-based compounds. To quantitatively describe the variation of CSW as a function of $T_{\mathrm{e}}$, we focus our analysis on the evaluation of the area of the symmetrized TR-ARPES EDCs (SEDCs) at $\mathbf{k}_{\mathrm{F}}$. In fact, provided the particle-hole symmetry of the spectral function-which has been experimentally verified at $\mathbf{k}_{\mathrm{F}}$ in the near-nodal region of Bi2212 [15,27] — SEDCs directly map onto the spectral function, $\operatorname{SEDC}(\omega) \propto \mathrm{A}\left(\mathbf{k}_{\mathrm{F}}, \omega\right)[28,29]$. Here, we define the relative suppression of coherent spectral weight, $\triangle \mathrm{CSW}$, as the difference between the integrated area under the SEDCs at $\mathbf{k}_{\mathrm{F}}$ at low and high $T_{\mathrm{e}}$ (i.e., before and after the pump excitation), in the $[-0.08,0.08] \mathrm{eV}$ range [see inset of Fig. 3(a)]. This approach makes our analysis bias-free from the choice of specific models or fitting procedures of the SEDCs. The obtained $\triangle \mathrm{CSW}$ is plotted directly as a function of $T_{\mathrm{e}}$ in Fig. 3 for different doping levels of Bi2212 and Bi2201. Despite the large number of dopings and related $T_{c}$ across the studied compounds, a remarkable similarity characterizes the $T$-dependent suppression of $\triangle \mathrm{CSW}$ in the explored $100 \mathrm{~K}$ temperature range. Most importantly, the decrease of $\Delta \operatorname{CSW}\left(T_{\mathrm{e}}\right)$ does not exhibit any modification-abrupt or otherwise-across the superconducting-to-normal state phase transitions, and continues for $T_{\mathrm{e}} \gg T_{c}$ (colored and gray bars in Fig. 3 highlight $T_{c}$ for the various dopings studied here; see Fig. 4(c) for data of Bi2212-OP91 above $T_{c}$ ). These findings demonstrate the absence of a direct contribution of the superconducting phase to $\Delta \mathrm{CSW}\left(T_{\mathrm{e}}\right)$, and establish the
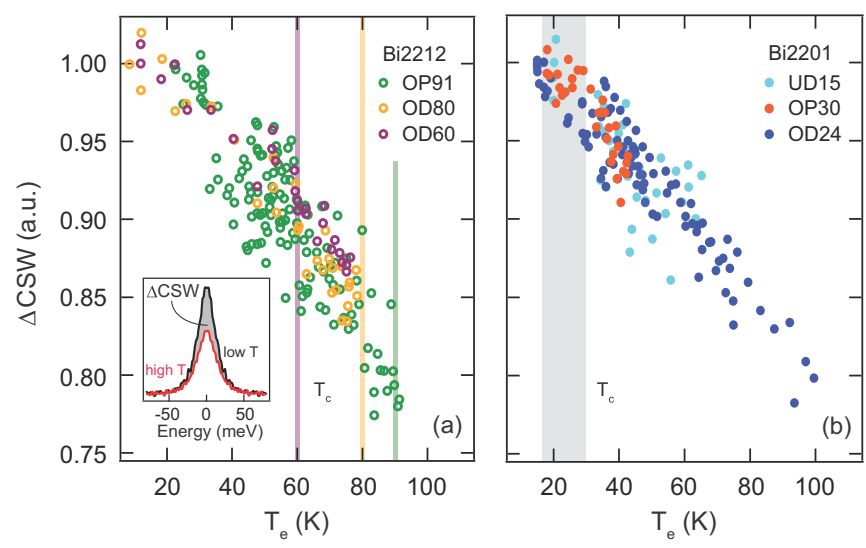

FIG. 3. (a) Relative variation of the nodal coherent spectral weight $\triangle \mathrm{CSW}$ as a function of the electronic temperature $T_{\mathrm{e}}$ tracked via TR-ARPES, for three different doping levels of Bi2212. The inset illustrates the difference in the integrated area of the symmetrized EDCs in the $[-0.08,0.08] \mathrm{eV}$ range, which defines $\triangle \mathrm{CSW}$. (b) Same as in (a), but for three doping levels of Bi2201. (a) Colored and (b) gray vertical bars mark the range of $T_{c}$ for the different compounds. The estimated temperatures at negative pump-probe-delays are listed in Appendix A for each sample.

$T$ dependence of the nodal coherent spectral weight as a ubiquitous behavior of Bi-based cuprates. The data reported in Fig. 3 also exclude a possible relation of the observed nodal suppression of CSW to the pseudogap phenomenon. In fact, despite no equilibrium pseudogap having been reported for the very overdoped Bi2212-OD60 compound [30-32], its $\Delta \mathrm{CSW}$ [purple open circles in Fig. 3(a)] is comparable to that observed for the other compounds.

Upon ruling out any dependence on the characteristic $T_{c}$, we explore the possible contribution of normal state properties to the $T$ dependence of CSW. In the following we demonstrate that the progressive meltdown of the nodal CSW can be understood in terms of the intrinsic $\omega$ and $T$ dependence of the self-energy within the FL model. The FL self-energy can be written as $[1,17]$

$$
\Sigma_{\mathrm{FL}}(\omega, T)=-\alpha \omega-i\left[\Gamma+\beta\left(\omega^{2}+\pi^{2} k_{B}^{2} T^{2}\right)\right],
$$

where $\alpha, \beta$, and $\Gamma$ are positive parameters. $\Gamma$ represents a scattering rate term associated with static impurities, and is thus independent of energy and temperature [33]. Using Eq. (1), we can calculate the $T$ dependence of the spectral function and compute the resulting $\Delta \mathrm{CSW}^{\mathrm{FL}}$. We emphasize that the discussion is limited to $\mathbf{k}=\mathbf{k}_{\mathrm{F}}$ (i.e., $\varepsilon_{\mathbf{k}}^{b}=0$ ), which allows for a direct comparison to the experimental data presented in Fig. 3. In order to estimate the values of $\alpha$ and $\beta$, and thus provide a more quantitative modeling of $\triangle \mathrm{CSW}^{\mathrm{FL}}$, we developed a global fit analysis of EDCs and MDCs in terms of Eq. (1). Figure 4(a) shows the results of this global fitting procedure for three different temperatures of Bi2212-OP91 and Bi2201-OD24. We assume a bare velocity $v_{0}=3.8(3.35) \mathrm{eV} \AA$ for $\mathrm{Bi} 2212$ (Bi2201), as reported from previous studies [34-36]. Note that the parameter $\alpha$ can be related to the bare velocity by the expression $\alpha=\frac{v_{0}}{v_{\mathrm{F}}}-1$, where $v_{\mathrm{F}}$ is the renormalized Fermi velocity. Despite several mechanisms playing a role in determining the self-energy, 

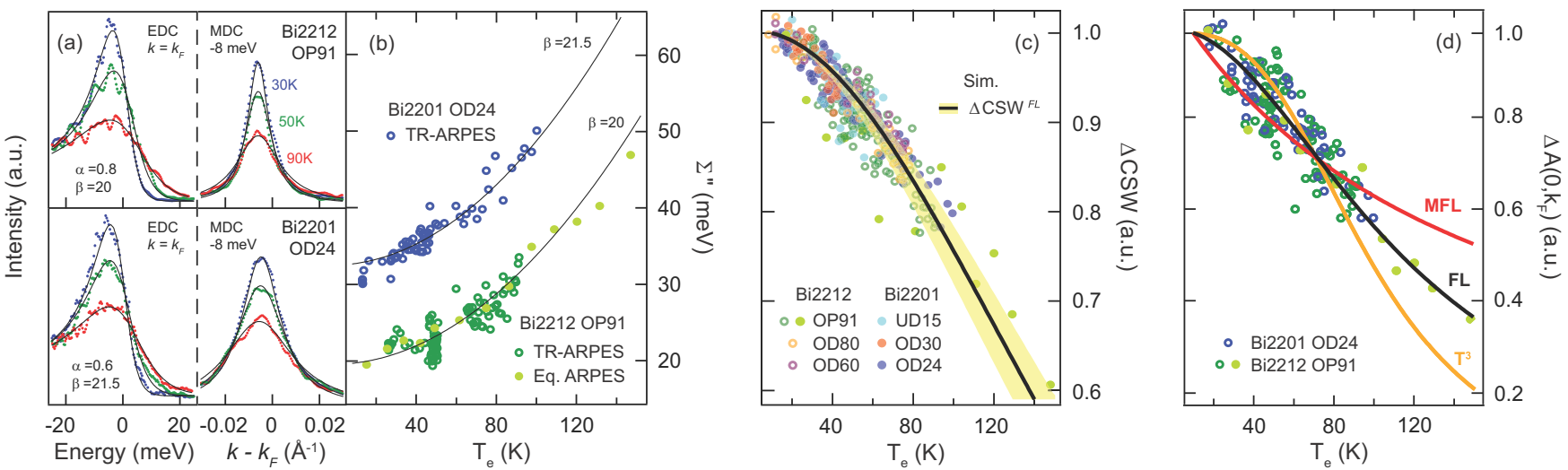

FIG. 4. (a) Nodal EDCs at $\mathbf{k}_{\mathrm{F}}$ and MDCs at $-8 \mathrm{meV}$ for Bi2212-OP91 and Bi2201-OD24, probed by TR-ARPES at different temperatures. Black solid lines represent the results of the EDC-MDC global fitting procedure using Eq. (1), as explained in the main text. Optimal fits to the data are found with $(\alpha=0.8, \beta=20)$ and $(\alpha=0.6, \beta=21.5)$ for Bi2212-OP91 and Bi2201-OD24, respectively. (b) Temperature evolution of the imaginary part of the electron self-energy extracted via fitting of MDCs at $-8 \mathrm{meV}$ (integration windows $\pm 5 \mathrm{meV}$ ) via a Lorentzian-like function for Bi2201-OD24 and Bi2212-OP91. The solid black lines are quadratic curves defined by the $\beta$ coefficients obtained via the global fit procedure of panel (a). (c) Comparison between the experimental CSW suppression (TR-ARPES data from Fig. 3; equilibrium ARPES for Bi2212-OP91 in full green circles) and the simulated $\Delta \mathrm{CSW}^{\mathrm{FL}}$ within the FL model via Eq. (1). The solid black line is computed for $\alpha=0.7$, $\beta=20.75, \Gamma=0.02$, while the yellow shading accounts for the parameters' range defined by the global fit. (d) Normalized temperature evolution of the spectral function $\triangle A\left(0, \mathbf{k}_{\mathrm{F}}\right)$, for Bi2201-OD24 (TR-ARPES data) and Bi2212-OP91 (TR- and equilibrium ARPES). The integration range in momentum and energy is $0.005 \AA^{-1}$ and $6 \mathrm{meV}$, respectively. The solid lines are best fits to the experimental data by using Eq. (2) for the different models with $\Gamma=0.02$ : FL (black, $\beta=20.35$ ), MFL (red, $\lambda=0.96$ ), and $T^{3}$ (orange, $\eta=3.28 \times 10^{4}$ ). All spectra have been deconvoluted from the energy resolution before extracting EDCs and MDCs in panels (a), (b), and (d).

in our analysis we do not differentiate the various contributions, and assume a linear renormalized dispersion in the first $0.2 \mathrm{eV}$ below $E_{\mathrm{F}}$. The best simultaneous global fit to EDCs and MDCs is achieved for $\alpha=0.8$ and $\beta=20(\alpha=0.6$ and $\beta=21.5$ ) for Bi2212-OP91 (Bi2201-OD24). These values are consistent with the band renormalization reported in previous ARPES studies [35,37,38], and capture the quadratic temperature dependence of the imaginary part of the selfenergy [see Fig. 4(b)], further validating our global fit results. We recognize that extracting the underlying functional form of the self-energy relying solely on the $\omega$ and/or $T$ evolution of the MDC width is extremely challenging, as evidenced by variance in the reported behaviours ranging from linear to cubic $[18,33-35,39]$. To this end, we emphasize that the EDC-MDC global fit procedure presented here was tested against a general functional form of the imaginary part of the self-energy, namely $\Sigma^{\prime \prime}(\omega, T)=-\Gamma-\beta\left(\omega^{2}+\pi^{2} k_{B}^{2} T^{2}\right)^{1 / a}$. While $a=1$ describes the FL self-energy, $a=2$ and $a=$ $2 / 3$ resemble the linear $\omega$ and $T$ dependence of the phenomenological MFL self-energy [18,33] and the cubic $T$ evolution of $\Sigma^{\prime \prime}[19,39]$, respectively. The global fit analysis consistently converges to $a=1 \pm 0.1$, pointing towards a description of the experimental data in terms of the FL model.

Having established an effective range for the parameters in Eq. (1), we now simulate the suppression of spectral weight $\triangle \mathrm{CSW}^{\mathrm{FL}}$ at $\mathbf{k}_{\mathrm{F}}$ (integration window $[-0.08,0.08] \mathrm{eV}$, normalized to the $10 \mathrm{~K}$ value; see Appendix $\mathrm{D}$ for the analytical expression of $\mathrm{CSW}^{\mathrm{FL}}$ ). This is plotted in Fig. 4(c) superimposed to the TR-ARPES experimental data shown in Fig. 3 and additional equilibrium ARPES data for Bi2201-OP91. In particular, $\alpha=0.7, \beta=20.75$, and $\Gamma=0.02$ were used to simulate the solid black line, while the yellow shading corresponds to the parameters' range defined by the global fit in Fig. 4(a). A remarkable agreement is observed between the FL simulation and the experimental suppression of CSW, both in terms of functional form and magnitude, with a quenching of the CSW as large as $\sim 25 \%$ at $100 \mathrm{~K}$. Despite the significant suppression of CSW at $\mathbf{k}_{\mathrm{F}}$, we note that the tomographic density of states along the nodal direction [40] (i.e., $\int_{-\infty}^{\infty} A(\bar{k}, \omega, T) d \bar{k}$, where $\bar{k}$ defines the $\bar{\Gamma}-\bar{Y}$ direction) does not vary within a $3 \%$ uncertainty over the explored temperature range, consistent with general spectral-function sum rules (more details in Appendix E).

In further support of our interpretation, we examine also the $T$ dependence of the spectral function at $\omega=0$, $A\left(0, \mathbf{k}_{\mathrm{F}}, T\right)$, which can be written as

$$
A\left(0, \mathbf{k}_{\mathrm{F}}, T\right)= \begin{cases}\frac{1}{\pi} \frac{1}{\Gamma+\beta\left(\pi k_{B} T\right)^{2}}, & \text { for FL, } \\ \frac{1}{\pi} \frac{1}{\Gamma+\lambda\left(\frac{\pi}{2} k_{B} T\right)}, & \text { for MFL, } \\ \frac{1}{\pi} \frac{1}{\Gamma+\eta\left(k_{B} T\right)^{3}}, & \text { for } T^{3},\end{cases}
$$

where $\Gamma$ is a scattering rate term independent of energy and temperature as in Eq. (1). We remark that the $T$ dependence in Eq. (2) is solely determined by the parameters $\beta, \lambda$, and $\eta$, offering a simpler and novel comparison with existing models. Figure 4(d) shows the relative variation of the spectral function, $\Delta A\left(0, \mathbf{k}_{\mathrm{F}}\right)$, for the three models of Eq. (2) (solid lines, normalized to the $10 \mathrm{~K}$ value) superimposed to its experimental counterpart for Bi2201-OD24 and Bi2212OP91. While the FL model captures remarkably well the experimental data, MFL and $T^{3}$ instead fail in reproducing the observed $T$ dependence over the entire explored range. This finding is consistent with optical and transport studies reporting evidences of a FL regime in the underdoped region 
of other high- $T_{c}$ cuprates [41-43], as well as with a recent ARPES study of an overdoped La-based cuprate, which reports a FL-to-MFL crossover moving from the nodal to the antinodal region [44]. We note that recent angle-dependent magnetoresistance measurements of Tl- and La-based compounds indicate the potential coexistence of quadratic and linear contributions to the scattering rate in either isotropic or anisotropic form [45,46]. As for the Bi-based cuprates, whether a deviation from the FL description reported here is observed upon moving from the nodal to the antinodal region remains an open question, requiring additional momentumresolved investigations.

\section{CONCLUSION}

In conclusion, we reported a comprehensive study of the meltdown of the nodal CSW as a function of the electronic temperature in Bi-based cuprates. By employing ARPES, both in its conventional and time-resolved fashion, we investigated various doping levels and compounds over a broad temperature range, and revealed a ubiquitous $T$ dependence of the nodal CSW bearing no direct relation to the superconducting or pseuodgap temperature scales. Instead, the observed suppression of the nodal CSW at $\mathbf{k}_{\mathrm{F}}$ naturally stems from the temperature and energy dependence of the FL electron self-energy. We remark that these conclusions were attained via a multianalysis approach which combines EDC-MDC global fitting and scattering rate analysis with a novel investigation of the $T$ evolution of the $\omega=0$, as well as energy-integrated, nodal coherent spectral weight. While our findings demonstrate the FL nature of quasiparticles along the gapless nodal direction of Bi-based cuprates, further investigations are needed to address whether such a scenario holds along the whole Fermi arc or if a crossover to a MFL or $T^{3}$ behavior occurs instead. To this end, the pump-probe approach presented here for the study of the CSW temperature evolution may soon be extended to the antinodal region of cuprates, owing to the advent of TR-ARPES in the extreme-ultra-violet regime $[47,48]$.

\section{ACKNOWLEDGMENTS}

We gratefully acknowledge E. Ostroumov for his technical support and P. D. Johnson for insightful discussions. This research was undertaken thanks, in part, to funding from the Max Planck-UBC-UTokyo Center for Quantum Materials and the Canada First Research Excellence Fund, Quantum Materials, and Future Technologies Program. This project is also funded by the Gordon and Betty Moore Foundation's EPiQS Initiative, Grant No. GBMF4779 to A.D. and D.J.J.; the Killam, Alfred P. Sloan, and Natural Sciences and Engineering Research Council of Canada's (NSERC's) Steacie Memorial Fellowships (A.D.); the Alexander von Humboldt Fellowship (A.D.); the Canada Research Chairs Program (A.D.); NSERC, Canada Foundation for Innovation (CFI); British Columbia Knowledge Development Fund (BCKDF); and the CIFAR Quantum Materials Program. E.R. acknowledges support from the Swiss National Science Foundation (SNSF)
Grant No. P300P2 164649. Part of the research described in this work was performed at the Canadian Light Source, a national research facility of the University of Saskatchewan, which is supported by CFI, NSERC, the National Research Council (NRC), the Canadian Institutes of Health Research (CIHR), the Government of Saskatchewan, and the University of Saskatchewan. We acknowledge Elettra Sincrotrone Trieste for providing access to its synchrotron radiation facilities. H.E. acknowledges support from the JSPS KAKENHI Grant No. JP19H05823. The work at BNL was supported by the US Department of Energy, office of Basic Energy Sciences, Contract No. DOE-sc0012704.

M.Z. and F.B. contributed equally to this work.

\section{APPENDIX A: MATERIALS AND METHODS}

$\mathrm{Bi}_{2} \mathrm{Sr}_{2-x} \mathrm{La}_{x} \mathrm{CuO}_{6+\delta} \quad(\mathrm{Bi} 2201)$ and $\mathrm{Bi}_{2} \mathrm{Sr}_{2} \mathrm{CaCu}_{2} \mathrm{O}_{8+\delta}$ (Bi2212) samples were aligned via Laue diffraction along the nodal $\bar{\Gamma}-\bar{Y}$ direction to avoid replica bands [49-51], and cleaved in a vacuum at pressures and base temperature lower than $8 \times 10^{-11}$ Torr and $20 \mathrm{~K}$, respectively. Equilibrium ARPES measurements were performed at the Quantum Materials Spectroscopy Centre (QMSC) beamline at the Canadian Light Source using $27 \mathrm{eV} \sigma$-polarized light, with energy and momentum resolutions better than $5.5 \mathrm{meV}$ and $0.007 \AA^{-1}$, respectively. Additional ARPES experiments were conducted at the BaDElPh endstation at the Elettra synchrotron with tunable photon energy in the range 7-30 eV. TR-ARPES data were acquired at the UBC-Moore Center for Ultrafast Quantum Matter by pumping and probing with $\sigma$-polarized $1.55 \mathrm{eV}$ and $6.2 \mathrm{eV}$ pulses, and with overall energy, momentum, and temporal resolution of $18 \mathrm{meV}$, $0.0025 \AA^{-1}$, and $250 \mathrm{fs}$, respectively [15], unless specified differently in the main text. All TR-ARPES experiments were performed at a base cryostat temperature of $10 \mathrm{~K}$; however, overall pump thermal effects have to be taken into account independently for each experiment. We estimate an approximate temperature for negative pump-probe delays as follow, for the different samples: $15 \mathrm{~K}$ and $25 \mathrm{~K}$ at low and high pump fluences, respectively $\left(12 \mu \mathrm{J} / \mathrm{cm}^{2}, \mathrm{LF} ; 90 \mu \mathrm{J} / \mathrm{cm}^{2}\right.$, $\mathrm{HF}$ ) for Bi2201-OD24; $12 \mathrm{~K}$ for Bi2201-OD30, LF; $15 \mathrm{~K}$ for Bi2201-UD15, LF; $25 \mathrm{~K}$ and $45 \mathrm{~K}$ for Bi2212-OP91, LF and $\mathrm{HF}$, respectively; $12 \mathrm{~K}$ for Bi2212-OD80, LF; $15 \mathrm{~K}$ for Bi2212-OD60, LF.

\section{APPENDIX B: PHOTON ENERGY DEPENDENCE OF THE BILAYER SPLITTING}

In an effort to compare the nodal quasiparticle lineshape, as well as the temperature dependence of the coherent spectral weight, as probed by different photon energies for Bi2212, it is important to verify matrix-elements-related effects. The two interacting $\mathrm{CuO}_{2}$ planes in $\mathrm{Bi} 2212$ lead to bilayer band splitting, e.g., two separate antibonding (AB) and bonding (B) states crossing $E_{F}[52,53]$. Therefore, different photon energies may probe either one of these states (or both equivalently), as shown in Fig. 5. In this work, we accurately selected $6.2 \mathrm{eV}$ and $27 \mathrm{eV}$ photon energies for time-resolved and equilibrium ARPES measurements, respectively, to have similar 


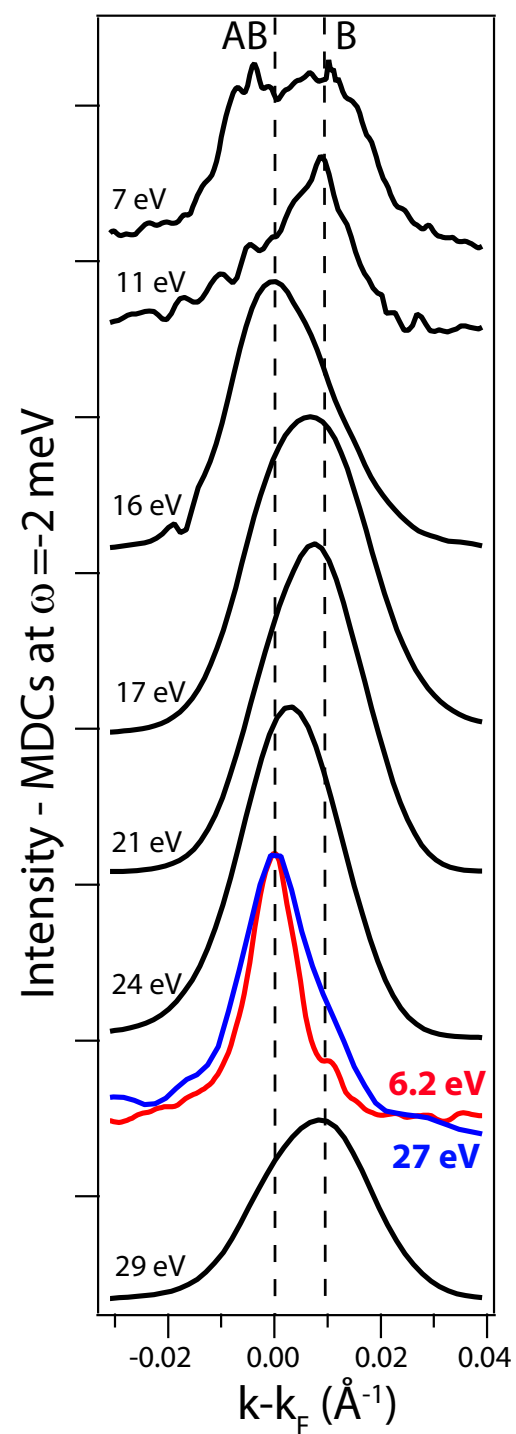

FIG. 5. Momentum distribution curves (MDCs) at $\omega=-2 \mathrm{meV}$ (5 meV integration window) along the nodal direction $\Gamma-\mathrm{Y}$ of Bi2212-OP91. Black curves have been acquired at the Elettra synchrotron, BaDElPh beamline; the blue curve has been acquired at the CLS, QMSC beamline, with $27 \mathrm{eV}$ photons; the red curve has been acquired at the UBC-Moore Center for Ultrafast Quantum Matter with $6.2 \mathrm{eV}$ photons. The blue and red curves represent the photon energies employed in this work. Vertical dashed lines highlight the separation between antibonding (AB) and bonding (B) states, which is approximately $0.01 \AA^{-1}$.

matrix-elements for Bi2212 compounds. In this specific case, with the selected photon energies we mainly probe the $\mathrm{AB}$ state, as shown in Fig. 5 by the blue and red lines.

\section{APPENDIX C: RULING OUT NONLINEAR EFFECTS IN THE PHOTOELECTRON DETECTION}

The nonlinear response of the photoelectron detection may affect a correct assessment of ARPES spectral features, especially when sharp and intense quasiparticle peaks occur along with a low level background, as often is the case of ARPES measurements on cuprates. This can result in an artificial suppression/enhancement of the quasiparticle peak, thus preventing the correct evaluation of small modifications of the coherent spectral weight (CSW). Postacquisition algorithms were developed to correct ARPES maps acquired in a nonlinear detection regime [26,54], however, such procedures may not perfectly compensate all nonlinear effects.

To avoid possible contributions of the detector's nonlinearity to the observed evolution of CSW, all the data presented in this work were acquired at an extremely low count-rate, in a regime benchmarked by spectra obtained in the singlepulse-counting (PC) mode, which is intrinsically linear at a low count-rate. Figures 6(a) and 6(b) show the response of the detector employed at the QMSC beamline for the equilibrium ARPES measurements (Scienta R4000) in the analog-to-digital converter (ADC) and PC mode, respectively; a clear deviation from the linear response develops in the ADC mode as the photon flux increases. In acquiring our equilibrium ARPES data, we therefore limited the maximum ADC count-rate to a value which ensures a linear response of the detector, as well as a quasiparticle lineshape equivalent to that probed via PC mode, as shown by the energy distribution curves (EDCs) in Fig. 6(c). Similarly, the maximum countrate employed for TR-ARPES measurements performed at UBC was limited to achieve an effective PC mode regime with both the spectrometers used in this work, namely a Scienta DA30 [data on Bi2212-OP91, see Fig. 6(d)] and a SPECS Phoibos 150 (data on all others compounds, not shown).

This approach rules out any suppression/enhancement of coherent spectral weight artificially induced by nonlinear effects in the photoelectron detection, while providing higher statistics and reduced acquisition time.

\section{APPENDIX D: ANALYTICAL FORM OF COHERENT SPECTRAL WEIGHT}

In the scenario of the Fermi liquid framework, i.e., using the self-energy of Eq. (1) in the main text, the full temperature dependence of the coherent spectral weight at the specific momentum $\mathbf{k}_{\mathrm{F}}, \mathrm{CSW}_{\mathrm{FL}}\left(\mathbf{k}_{\mathrm{F}}, T\right)=\int_{-\infty}^{\infty} A\left(\mathbf{k}_{\mathrm{F}}, \omega, T\right) d \omega$, can be expressed analytically via application of the residue theorem

$$
\operatorname{CSW}_{\mathrm{FL}}\left(\mathbf{k}_{\mathrm{F}}, T\right)=\frac{2}{\sqrt{(1+\alpha)^{2}+4 \beta^{2}\left[\Gamma / \beta+\left(\pi k_{B} T\right)^{2}\right]}} .
$$

By visual inspection of Eq. (D1), it is evident that $\operatorname{CSW}\left(\mathbf{k}_{\mathrm{F}}, T\right)$ depends on the temperature $T$, as directly shown by the experimental data in Fig. 3 and Fig. 4(a) to 4(c).

\section{APPENDIX E: NODAL TOMOGRAPHIC DENSITY OF STATES}

Even if the nodal coherent spectral weight at $\mathbf{k}_{\mathrm{F}}$ decreases as a function of the increasing temperature, the nodal tomographic density of states (TDOS) along the cut perpendicular to the Fermi surface [40] does not vary within a 3\% uncertainty. Here we provide more experimental evidences in support of this claim for Bi2212-OP91.

The nodal TDOS can be expressed as $\int_{-\infty}^{\infty} A(\bar{k}, \omega, T) d \bar{k}$, with $\bar{k}$ representing the $\bar{\Gamma}-\bar{Y}$ direction. To remove spurious 

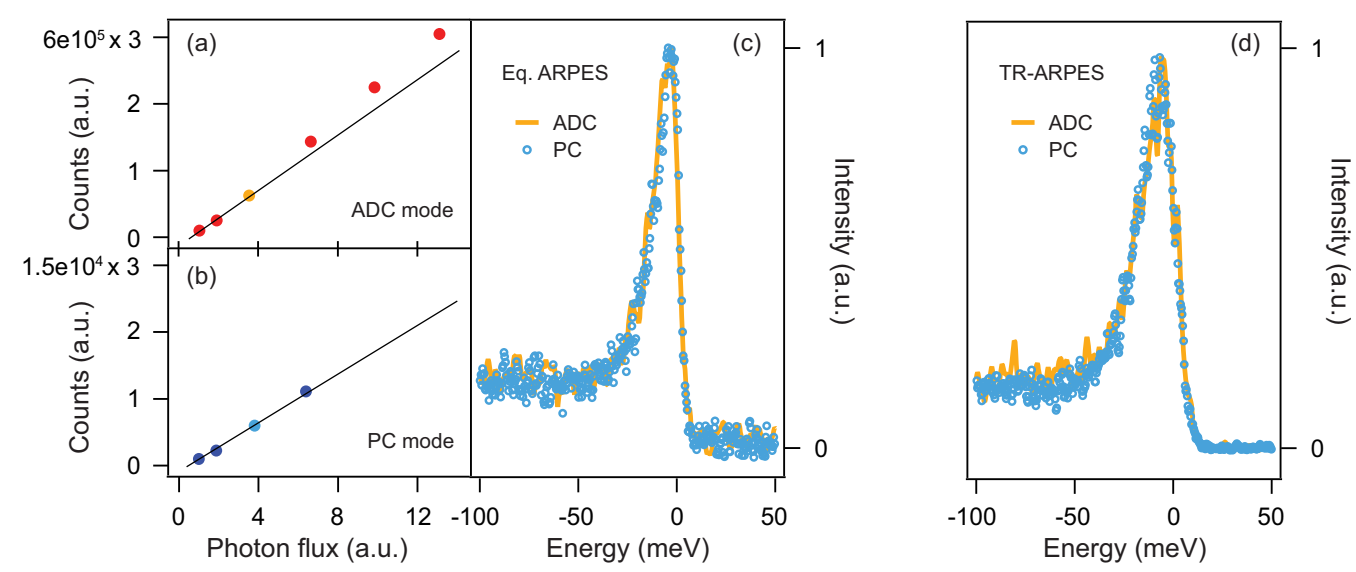

FIG. 6. (a, b) Characterization of the response of the Scienta R4000 analyzer at the QMSC beamline: count-rate under the quasiparticle peak in the analog-to-digital converter (ADC) and single-pulse-counting (PC) detection mode, respectively. The orange and light blue markers indicate the maximum count-rate employed in this work. (c) Comparison between nodal EDCs at $\mathbf{k}_{\mathrm{F}}$ of Bi2212-OP91 acquired at the QMSC beamline with ADC (orange line) and PC (light blue markers) mode at the maximum count-rate. Temperature $20 \mathrm{~K}$ and photon energy $27 \mathrm{eV}$. (d) Nodal EDCs at $\mathbf{k}_{\mathrm{F}}$ of Bi2212-OP91 acquired with the TR-ARPES setup at UBC using a Scienta DA30 analyzer in the two different detection modes. Temperature $10 \mathrm{~K}$ and photon energy $6.2 \mathrm{eV}$. All EDCs shown here have not been deconvoluted for the energy resolution broadening.

background contributions to the experimental spectral weight (such as incoherent spectral weight, secondary electrons, and electrons scattered in the detection process), and thus achieving a precise evaluation of the temperature evolution of the nodal TDOS, we applied the following procedure. First, we fit the time-resolved nodal momentum distribution curves (MDCs) with a Lorentzian-like function defined by only four parameters: peak position, width, area, and offset. This last accounts for all noncoherent/background contributions to the spectral weight and it can be subsequently subtracted from the ARPES nodal map. Once these contributions are removed, the experimental spectral weight is integrated in momentum $\left(-0.1 \AA^{-1}<\bar{k}<0.1 \AA^{-1}\right)$, as shown in Fig. 7(a). Given that the ARPES intensity is directly proportional to both the spectral function and electronic distribution, Fig. 7(a) resembles the TDOS multiplied by the Fermi-Dirac distribution for any pump-probe delay, i.e., any transient electronic temperature $T_{e}$ [see right panel Fig. 7(a)]. To average out thermal broadening effects, i.e., Fermi-Dirac broadening, we integrate in energy symmetrically across $E_{F}$ in the region relevant to the quasiparticle peak $(-40 \mathrm{meV}<\omega<40 \mathrm{meV}$; note that for $T=120 \mathrm{~K}, k_{B} T \approx 10 \mathrm{meV}$ ). Figure 7 (b) shows the evolution of this $\omega$-integrated nodal TDOS at $E_{F}$ as a function of the transient electronic temperature extracted for Bi2212-OP91, where values have been normalized to that of the lowest temperature. Only a very limited variation $(<3 \%$, orange shaded area) is observed over the entire $100 \mathrm{~K}$ temperature range.
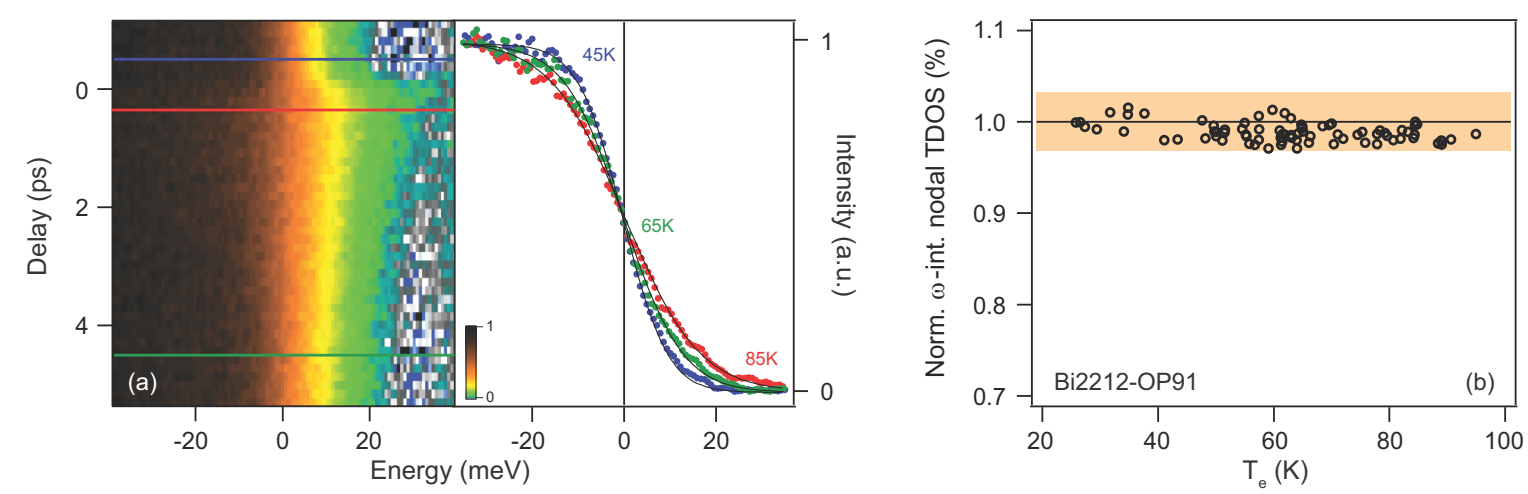

FIG. 7. (a) Momentum-integrated TR-ARPES map along the nodal direction of Bi2212-OP91 as a function of the pump-probe delay (integration window $\left[-0.1 \AA^{-1}, 0.1 \AA^{-1}\right]$ about $\mathbf{k}_{\mathrm{F}}$; high fluence data). Noncoherent/background contributions to the spectral weight have been subtracted as explained in the text. Right panel: Momentum integrated energy distribution curves for selected pump-probe delays; black lines are fits to Fermi-Dirac like functions. (b) $\omega$-integrated nodal TDOS of Bi2212-OP91 as a function of the effective electronic temperature, normalized to the lowest temperature value. The momentum and energy integration windows are $\left[-0.1 \AA^{-1}, 0.1 \AA^{-1}\right]$ and $[-40 \mathrm{meV}, 40 \mathrm{meV}]$, respectively. The orange shaded area highlights variations of $\pm 3 \%$. 
[1] A. Damascelli, Z. Hussain, and Z.-X. Shen, Rev. Mod. Phys. 75, 473 (2003).

[2] R. Comin and A. Damascelli, Annu. Rev. Condens. Matter Phys. 7, 369 (2016).

[3] B. Keimer, S. A. Kivelson, M. R. Norman, J. Uchida, and S. Zaanen, Nature 518, 179 (2015).

[4] A. Frano, S. Blanco-Canosa, B. Keimer, and R. J. Birgeneau, J. Phys.: Condens. Matter 32, 374005 (2020).

[5] G. A. Sawatzky, Nature 342, 480 (1989).

[6] Z.-X. Shen and G. A. Sawatzky, Physica Status Solidi (b) 215, 523 (1999).

[7] H. Ding, M. R. Norman, J. C. Campuzano, M. Randeria, A. F. Bellman, T. Yokoya, T. Takahashi, T. Mochiku, and K. Kadowaki, Phys. Rev. B 54, R9678 (1996).

[8] D. L. Feng, D. H. Lu, K. M. Shen, C. Kim, H. Eisaki, A. Damascelli, R. Yoshizaki, J.-I. Shimoyama, K. Kishio, G. D. Gu, S. Oh, A. Andrus, J. O'Donnell, J. N. Eckstein, and Z.-X. Shen, Science 289, 277 (2000).

[9] H. Ding, J. R. Engelbrecht, Z. Wang, J. C. Campuzano, S.-C. Wang, H.-B. Yang, R. Rogan, T. Takahashi, K. Kadowaki, and D. G. Hinks, Phys. Rev. Lett. 87, 227001 (2001).

[10] A. Lanzara, P. V. Bogdanov, X. J. Zhou, S. A. Kellar, D. L. Feng, E. D. Lu, T. Yoshida, H. Eisaki, A. Fujimori, K. Kishio, J.-I. Shimoyama, T. Noda, S. Uchida, Z. Hussain, and Z.-X. Shen, Nature 412, 510 (2001).

[11] P. D. Johnson, T. Valla, A. V. Fedorov, Z. Yusof, B. O. Wells, Q. Li, A. R. Moodenbaugh, G. D. Gu, N. Koshizuka, C. Kendziora, S. Jian, and D. G. Hinks, Phys. Rev. Lett. 87, 177007 (2001).

[12] D. Fournier, G. Levy, Y. Pennec, J. L. McChesney, A. Bostwick, E. Rotenberg, R. Liang, W. N. Hardy, D. A. Bonn, I. S. Elfimov, and A. Damascelli, Nat. Phys. 6, 905 (2010).

[13] J. Graf, C. Jozwiak, C. L. Smallwood, H. Eisaki, R. A. Kaindl, D.-H. Lee, and A. Lanzara, Nat. Phys. 7, 805 (2011).

[14] C. L. Smallwood, W. Zhang, T. L. Miller, C. Jozwiak, H. Eisaki, D.-H. Lee, and A. Lanzara, Phys. Rev. B 89, 115126 (2014).

[15] F. Boschini, E. H. da Silva Neto, E. Razzoli, M. Zonno, S. Peli, R. P. Day, M. Michiardi, M. Schneider, B. Zwartsenberg, P. Nigge, R. D. Zhong, J. Schneeloch, G. D. Gu, S. Zhdanovich, A. K. Mills, G. Levy, D. J. Jones, C. Giannetti, and A. Damascelli, Nat. Materials 17, 416 (2018).

[16] Y. Zheng, X. Zhang, W. Zhou, X. Liu, and H. Zhao, Phys. Lett. A 381, 2731 (2017).

[17] D. Pines and P. Noziures, The Theory of Quantum Liquids V.1: Normal Fermi Liquids (Benjamin, Amsterdam, 1966).

[18] C. M. Varma, P. B. Littlewood, S. Schmitt-Rink, E. Abrahams, and A. E. Ruckenstein, Phys. Rev. Lett. 63, 1996 (1989).

[19] T. Dahm, P. J. Hirschfeld, D. J. Scalapino, and L. Zhu, Phys. Rev. B 72, 214512 (2005).

[20] H.-B. Yang, J. D. Rameau, P. D. Johnson, T. Valla, A. Tsvelik, and G. D. Gu, Nature 456, 77 (2008).

[21] E. Razzoli, G. Drachuck, A. Keren, M. Radovic, N. C. Plumb, J. Chang, Y.-B. Huang, H. Ding, J. Mesot, and M. Shi, Phys. Rev. Lett. 110, 047004 (2013).

[22] F. Cilento, G. Manzoni, A. Sterzi, S. Peli, A. Ronchi, A. Crepaldi, F. Boschini, C. Cacho, R. Chapman, E. Springate, H. Eisaki, G. Martin, M. Berciu, A. F. Kemper, A. Damascelli, M. Capone, C. Giannetti, and F. Parmigiani, Sci. Adv. 4, eaar1998 (2018).

[23] C. Giannetti, M. Capone, D. Fausti, M. Fabrizio, F. Parmigiani, and D. Mihailovic, Adv. Phys. 65, 58 (2016).
[24] F. Boschini, M. Zonno, E. Razzoli, R. P. Day, M. Michiardi, B. Zwartsenberg, P. Nigge, M. Schneider, E. H. da Silva Neto, A. Erb, S. Zhdanovich, A. K. Mills, G. Levy, C. Giannetti, D. J. Jones, and A. Damascelli, npj Quantum Mater. 5, 6 (2020).

[25] J. D. Koralek, J. F. Douglas, N. C. Plumb, Z. Sun, A. V. Fedorov, M. M. Murnane, H. C. Kapteyn, S. T. Cundiff, Y. Aiura, K. Oka, H. Eisaki, and D. S. Dessau, Phys. Rev. Lett. 96, 017005 (2006).

[26] T. J. Reber, N. C. Plumb, J. A. Waugh, and D. S. Dessau, Rev. Sci. Instrum. 85, 043907 (2014).

[27] H. Matsui, T. Sato, T. Takahashi, S.-C. Wang, H.-B. Yang, H. Ding, T. Fujii, T. Watanabe, and A. Matsuda, Phys. Rev. Lett. 90, 217002 (2003).

[28] M. R. Norman, H. Ding, M. Randeria, J. C. Campuzano, T. Yokoya, T. Takeuchi, T. Takahashi, T. Mochiku, K. Kadowaki, P. Guptasarma, and D. G. Hinks, Nature 392, 157 (1998).

[29] S. Parham, H. Li, T. J. Nummy, J. A. Waugh, X. Q. Zhou, J. Griffith, J. Schneeloch, R. D. Zhong, G. D. Gu, and D. S. Dessau, Phys. Rev. X 7, 041013 (2017).

[30] S. Kawasaki, C. Lin, P. L. Kuhns, A. P. Reyes, and G.-Q. Zheng, Phys. Rev. Lett. 105, 137002 (2010).

[31] I. M. Vishik, M. Hashimoto, R.-H. He, W.-S. Lee, F. Schmitt, D. Lu, R. G. Moore, C. Zhang, W. Meevasana, T. Sasagawa, S. Uchida, K. Fujita, S. Ishida, M. Ishikado, Y. Yoshida, H. Eisaki, Z. Hussain, T. P. Devereaux, and Z.-X. Shen, Proc. Natl Acad. Sci. 109, 18332 (2012).

[32] U. Chatterjee, D. Ai, J. Zhao, S. Rosenkranz, A. Kaminski, H. Raffy, Z. Li, K. Kadowaki, M. Randeria, M. R. Norman, and J. C. Campuzano, Proc. Natl Acad. Sci. 108, 9346 (2011).

[33] E. Abrahams and C. M. Varma, Proc. Natl Acad. Sci. 97, 5714 (2000).

[34] A. Kaminski, H. M. Fretwell, M. R. Norman, M. Randeria, S. Rosenkranz, U. Chatterjee, J. C. Campuzano, J. Mesot, T. Sato, T. Takahashi, T. Terashima, M. Takano, K. Kadowaki, Z. Z. Li, and H. Raffy, Phys. Rev. B 71, 014517 (2005).

[35] A. A. Kordyuk, S. V. Borisenko, A. Koitzsch, J. Fink, M. Knupfer, and H. Berger, Phys. Rev. B 71, 214513 (2005).

[36] W. Meevasana, F. Baumberger, K. Tanaka, F. Schmitt, W. R. Dunkel, D. H. Lu, S.-K. Mo, H. Eisaki, and Z.-X. Shen, Phys. Rev. B 77, 104506 (2008).

[37] X. J. Zhou, T. Yoshida, A. Lanzara, P. V. Bogdanov, S. A. Kellar, K. M. Shen, W. L. Yang, F. Ronning, T. Sasagawa, T. Kakeshita, T. Noda, H. Eisaki, S. Uchida, C. T. Lin, F. Zhou, J. W. Xiong, W. X. Ti, Z. X. Zhao, A. Fujimori, Z. Hussain, and Z.-X. Shen, Nature 423, 398 (2003).

[38] W. Zhang, C. Hwang, C. L. Smallwood, T. L. Miller, G. Affeldt, K. Kurashima, C. Jozwiak, H. Eisaki, T. Adachi, Y. Koike, D.-H. Lee, and A. Lanzara, Nat. Commun. 5, 4959 (2014).

[39] T. Valla, T. E. Kidd, J. D. Rameau, H.-J. Noh, G. D. Gu, P. D. Johnson, H.-B. Yang, and H. Ding, Phys. Rev. B 73, 184518 (2006).

[40] T. J. Reber, N. C. Plumb, Z. Sun, Y. Cao, Q. Wang, K. McElroy, H. Iwasawa, M. Arita, J. S. Wen, Z. J. Xu, G. Gu, Y. Yoshida, H. Eisaki, Y. Aiura, and D. S. Dessau, Nat. Phys. 8, 606 (2012).

[41] S. I. Mirzaei, D. Stricker, J. N. Hancock, C. Berthod, A. Georges, E. van Heumen, M. K. Chan, X. Zhao, Y. Li, M. Greven, N. Barišić, and D. van der Marel, Proc. Natl Acad. Sci. 110, 5774 (2013). 
[42] N. Barišić, M. K. Chan, Y. Li, G. Yu, X. Zhao, M. Dressel, A. Smontara, and M. Greven, Proc. Natl Acad. Sci. 110, 12235 (2013).

[43] C. Proust, B. Vignolle, J. Levallois, S. Adachi, and N. E. Hussey, Proc. Natl Acad. Sci. 113, 13654 (2016).

[44] J. Chang, M. Månsson, S. Pailhès, T. Claesson, O. J. Lipscombe, S. M. Hayden, L. Patthey, O. Tjernberg, and J. Mesot, Nat. Commun. 4, 2559 (2013).

[45] M. Abdel-Jawad, M. P. Kennett, L. Balicas, A. Carrington, A. P. Mackenzie, R. H. McKenzie, and N. E. Hussey, Nat. Phys. 2, 821 (2006).

[46] G. Grissonnanche, Y. Fang, A. Legros, S. Verret, F. Laliberté, C. Collignon, J. Zhou, D. Graf, P. Goddard, L. Taillefer, and B. J. Ramshaw, arXiv:2011.13054.

[47] A. K. Mills, S. Zhdanovich, M. X. Na, F. Boschini, E. Razzoli, M. Michiardi, A. Sheyerman, M. Schneider, T. J. Hammond, V. Süss, C. Felser, A. Damascelli, and D. J. Jones, Rev. Sci. Instrum. 90, 083001 (2019).

[48] M. X. Na, A. K. Mills, F. Boschini, M. Michiardi, B. Nosarzewski, R. P. Day, E. Razzoli, A. Sheyerman, M. Schneider, G. Levy, S. Zhdanovich, T. P. Devereaux, A. F. Kemper, D. J. Jones, and A. Damascelli, Science 366, 1231 (2019).
[49] P. D. C. King, J. A. Rosen, W. Meevasana, A. Tamai, E. Rozbicki, R. Comin, G. Levy, D. Fournier, Y. Yoshida, H. Eisaki, K. M. Shen, N. J. C. Ingle, A. Damascelli, and F. Baumberger, Phys. Rev. Lett. 106, 127005 (2011).

[50] N. L. Saini, J. Avila, A. Biaconi, A. Lanzara, M. C. Asensio, S. Tajima, G. D. Gu, and N. Koshizuka, Phys. Rev. Lett. 79, 3467 (1997).

[51] W. L. Yang, H. H. Wen, Y. M. Ni, J. W. Xiong, H. Chen, C. Dong, F. Wu, Y. L. Qin, and Z. X. Zhao, Physica C: Superconductivity 308, 294 (1998).

[52] D. L. Feng, N. P. Armitage, D. H. Lu, A. Damascelli, J. P. Hu, P. Bogdanov, A. Lanzara, F. Ronning, K. M. Shen, H. Eisaki, C. Kim, Z.-X. Shen, J.-I. Shimoyama, and K. Kishio, Phys. Rev. Lett. 86, 5550 (2001).

[53] H. Iwasawa, J. F. Douglas, K. Sato, T. Masui, Y. Yoshida, Z. Sun, H. Eisaki, H. Bando, A. Ino, M. Arita, K. Shimada, H. Namatame, M. Taniguchi, S. Tajima, S. Uchida, T. Saitoh, D. S. Dessau, and Y. Aiura, Phys. Rev. Lett. 101, 157005 (2008).

[54] C. L. Smallwood, J. P. Hinton, C. Jozwiak, W. Zhang, J. D. Koralek, H. Eisaki, D.-H. Lee, J. Orenstein, and A. Lanzara, Science 336, 1137 (2012). 